\title{
Comparison of fluorescent intercalating dyes for quantitative loop-mediated isothermal amplification (qLAMP)
}

Igor P. Oscorbin ${ }^{1,2}$, Ekaterina A. Belousova ${ }^{1}$, Aleksandr I. Zakabunin ${ }^{1}$, Ulyana A. Boyarskikh¹, and Maksim L. Filipenko ${ }^{1,2}$

${ }^{1}$ Institute of Chemical Biology and Fundamental Medicine, Siberian Branch of the Russian Academy of Sciences, Novosibirsk, Russia and ${ }^{2}$ Novosibirsk State University, Novosibirsk, Russian Federation

BioTechniques 61:20-25 (July 2016) doi 10.2144/000114432

Keywords: LAMP; isothermal amplification; SYTO-9; SYTO-13; SYTO-82; SYBR Green I; SYBR Gold; EvaGreen; Bst DNA polymerase

Real-time or quantitative loop-mediated isothermal amplification (qLAMP) is a promising technique for the accurate detection of pathogens in organisms and the environment. Here we present a comparative study of the performance of six fluorescent intercalating dyes - SYTO-9, SYTO-13, SYTO82, SYBR Green I, SYBR Gold, EvaGreen - in three different qLAMP model systems. SYTO-9 and SYTO-82, which had the best results, were used for additional enzyme and template titration studies. SYTO-82 demonstrated the best combination of time-to-threshold $\left(T_{t}\right)$ and signal-to-noise ratio (SNR).

Over the last several decades, various methods for the isothermal amplification of nucleic acids have been developed. These techniques, including nucleic acid sequence-based amplification (NASBA) (1), rolling circle amplification (RCA) (2), loop-mediated isothermal amplification (LAMP) (3), and others (4-6), are valuable diagnostic tools, especially for in-field or point-of-care analyses. Among these different methods, LAMP has several major advantages, such as a high tolerance for inhibitors. This property may be useful not only for fast laboratory diagnostics but also for medical applications such as pointof-care testing where it is not possible to obtain highly purified nucleic acid samples (7). Another major benefit of LAMP is that it depends on isothermal reaction conditions, allowing the amplification of nucleic acids without the need for expensive laboratory equipment such as thermocyclers. The simplest configuration for performing LAMP requires only a heat source. In this case, the results can be observed with the naked eye. However, laboratories frequently utilize real-time measurement of product amplification for optimizing analysis conditions or quantifying templates. Using more complex equipment and additional reagents allows LAMP to be performed in a quantitative real-time format: quantitative LAMP (qLAMP).

An important characteristic of quantitative amplification methods is the real-time detection of reaction product accumulation. Measurement of turbidity, fluorescence, ion concentrations, and color can be used for visualization during qLAMP (8-11). In the case of fluorescence, several types of specific probes, intercalating dyes, and calcein have been suggested for detection (12-16). While fluorescent dyes have been compared directly for quantitative PCR (qPCR) $(17,18)$, only a few studies have compared the use of different intercalating dyes in qLAMP (19). This lack of information complicates the choice of an optimal fluorescent dye for qLAMP, hindering the use of LAMP in a real-time manner.

Here we compare six fluorescent dyes, most of which have already been used for qLAMP: SYBR Green I (9), SYBR
Gold, SYTO-9 (20), SYTO-13, SYTO-82 (21), and EvaGreen (19). Model systems for LAMP using lambda phage, Escherichia coli, and tick-borne encephalitis virus (TBEV) DNA were used for comparative testing. Titrations of dyes, enzyme, and template in qLAMP were performed and analyzed.

\section{Materials and methods}

DNA purification and

standards preparation

Lambda phage DNA was purchased from SibEnzyme (Novosibirsk, Russia). E. coli genomic DNA was isolated (22) from the JM109 strain.

The TBEV genome fragment was amplified using TBEV-F3/B3 primers (Table 1) and cDNA from infected Ixodes persulcatus as template and then cloned into the pCR 2.1 vector (Invitrogen, Waltham, MA) using the TA Cloning Kit according to manufacturer's instructions. The resulting plasmid pCR-TBEV was purified and quantified using a NanoDrop Lite A4 spectrophotometer (Thermo Fisher Scientific, Waltham, MA).

\section{METHOD SUMMARY}

Here we present a comparison of different fluorescent dyes for quantitative LAMP detection. Our data indicate that SYTO-82 is the best choice of dye for optimal qLAMP performance in terms of signal-to-noise ratio (SNR) and absence of inhibition. 
Serial dilutions (10-fold) of template in $1 \mathrm{ng} / \mathrm{\mu L}$ poly-dA DNA carrier were prepared for qLAMP sensitivity analysis.

\section{Quantitative LAMP}

Three different LAMP model systems were used for comparison of the fluorescent dyes. Distances between the F3/B3 primers, optimal temperatures, and GC-content of the amplicons are listed in Table 1.

LAMP reactions were performed in a final volume of $20 \mu \mathrm{L}$, which contained $2 \mu \mathrm{L}$ 10x ThermoPol Reaction Buffer [200 mM Tris- $\mathrm{HCl}, 100 \mathrm{mM}\left(\mathrm{NH}_{4}\right)_{2} \mathrm{SO}_{4}$, $100 \mathrm{mM} \mathrm{KCl}, 20 \mathrm{mM} \mathrm{MgSO}_{4}$, 1\% Triton $\mathrm{X}-100, \mathrm{pH}$ 8.8] (New England BioLabs, Ipswich, MA), $4.0 \mu \mathrm{M}$ of each dNTP (Biosan, Novosibirsk, Russia), $8 \cup$ of Bst DNA polymerase (New England BioLabs), 1.6 $\mu \mathrm{M}$ FIP and BIP primers, $0.8 \mu \mathrm{M} \mathrm{LF}$ and LB primers, and $0.2 \mu \mathrm{M}$ F3 and B3 primers (Biosan) (Table 1), according to the template. The reactions contained $1 \mathrm{ng}$ of lambda phage DNA (11), $1.6 \mathrm{ng}$ of $E$. coli genomic DNA (23), and $10^{4}$ copies of PCR-TBEV plasmid (24), unless otherwise indicated. Fluorescent dyes were purchased from Invitrogen (SYTO-9, SYTO-13, SYTO-82, SYBR Green I, SYBR Gold) or Biotium (Hayward, CA) (EvaGreen).

No-template control reactions contained Milli-Q water instead of template DNA.
Dye concentrations were calculated based on the stock solution concentrations provided by the supplier, with the exception of EvaGreen, the molar concentration of which could not be calculated as the dye's chemical structure is not publicly available. For SYBR Green I solution, 1× equals $2 \mu \mathrm{M}$ (25). Stock solutions of dyes $(200 \mu \mathrm{M}$; 100x in the case of EvaGreen) were prepared in Milli-Q water to minimize the effect of DMSO on qLAMP.

Assays were carried out in a CFX96 Touch (Bio-Rad Laboratories, Hercules, CA) at $57^{\circ} \mathrm{C}$ (TBEV), $59^{\circ} \mathrm{C}$ (lambda phage) and $60^{\circ} \mathrm{C}$ (E. coli) for $90 \mathrm{~min}$; the fluorescence signal was recorded at 1 min intervals in the FAM (SYTO-9, SYTO-13, SYBR Green I, SYBR Gold, EvaGreen) or HEX (SYTO-82) channels. Final fluorescence was measured with a CFX96 Touch Real-Time PCR Detection System (Bio-Rad Laboratories). Each experiment was done in triplicate.

The inhibitory effect of the dyes was analyzed by varying dye concentrations from $0.25-16 \mu \mathrm{M}$, keeping all other conditions unchanged.

LAMP reactions with different enzyme amounts (0.062-8 U/reaction) were performed with $1 \mu \mathrm{M}$ of dye $(0.5 x$ for EvaGreen).

LAMP sensitivity was investigated using serial 10 -fold dilutions of the templates (lambda phage DNA at $10^{7}-5$ copies/reaction; E. coli at $10^{6}-5$

Table 1. Parameters and primers for loop-mediated isothermal amplification (LAMP) model systems. $\begin{array}{llll}\text { Model system } & \text { Distance between F3/B3 primers (bp) } \quad \text { GC content (\%) } & \text { Optimal temperature }\left({ }^{\circ} \mathbf{C}\right)\end{array}$

\begin{tabular}{|c|c|c|c|}
\hline Lambda phage & 223 & 56.1 & 59 \\
\hline E. coli & 208 & 53.8 & 60 \\
\hline TBEV & 198 & 57.7 & 57 \\
\hline Primer & \multicolumn{3}{|c|}{$5^{\prime}-3^{\prime}$ sequence } \\
\hline Lambda-F3 & \multicolumn{3}{|c|}{ GAATGCCCGTTCTGCGAG } \\
\hline Lambda-B3 & \multicolumn{3}{|c|}{ TTCAGTTCCTGTGCGTCG } \\
\hline Lambda-LF & \multicolumn{3}{|c|}{ GGCGGCAGAGTCATAAAGCA } \\
\hline Lambda-LB & \multicolumn{3}{|c|}{ GGCAGATCTCCAGCCAGGAACTA } \\
\hline Lambda-FIP & \multicolumn{3}{|c|}{ CAGCATCCCTTTCGGCATACCAGGTGGCAAGGGTAATGAGG } \\
\hline Lambda-BIP & \multicolumn{3}{|c|}{ GGAGGTTGAAGAACTGCGGCAGTCGATGGCGTTCGTACTC } \\
\hline Ecoli-F3 & \multicolumn{3}{|c|}{ CTGCTGGGTGGTCAGGTA } \\
\hline Ecoli-B3 & \multicolumn{3}{|c|}{ GGATTTTCGCTTCCCACTCT } \\
\hline Ecoli-LF & \multicolumn{3}{|c|}{ TGTCGATTTGTTCAGGAACA } \\
\hline Ecoli-LB & \multicolumn{3}{|c|}{ CGACGACACTCCGATCGTT } \\
\hline Ecoli-FIP & \multicolumn{3}{|c|}{ AGCAGCTCTTCGTCATCAACCCAGGCGTTCCGTACATCATCG } \\
\hline Ecoli-BIP & \multicolumn{3}{|c|}{ TGTCTCAGTACGACTTCCCGGGCGCTTTCAGAGCAGAACCAC } \\
\hline TBEV-F3 & \multicolumn{3}{|c|}{ ACCATAAATGCCGACTGTGA } \\
\hline TBEV-B3 & \multicolumn{3}{|c|}{ TGCCACCACCATTGAGC } \\
\hline TBEV-LF & \multicolumn{3}{|c|}{ ACCTTGCCACTCTCTGTGG } \\
\hline TBEV-LB & \multicolumn{3}{|c|}{ ACGGGGACAGACTGTTGGTATGC } \\
\hline TBEV-FIP & \multicolumn{3}{|c|}{ CGGCAGCACCACTCTGGAATTCGGGGGCTTCTGTGAGGA } \\
\hline
\end{tabular}

copies/reaction; TBEV at $10^{6}-5$ copies/ reaction), $8 \cup$ of Bst DNA polymerase per reaction, and $1 \mu \mathrm{M}$ of the dyes.

\section{Data analysis}

Time-to-threshold $\left(T_{t}\right)$ was calculated using manual baseline settings in CFX Manager Software (Bio-Rad Laboratories). For characterization of the inhibitory effect, $T_{t}$ values were plotted against the dye concentration, and the linear range of the resulting curves was used for further calculation of the slopes of the lines, indicating the degree of inhibition.

Raw fluorescent data converted to relative fluorescent units (RFUs) were used to calculate signal-to-noise ratio (SNR). The SNR was defined as the ratio of RFUs in the reaction containing DNA relative to the reaction without DNA (no-template control) after the completion of the reactions (26).

\section{Results and discussion}

Our goal was to systematically compare a set of fluorescent dyes for use in qLAMP. First, the fluorescent dyes were titrated, keeping all other conditions unchanged. At this stage, the $T_{t}$ and SNR were determined in order to choose optimal dye concentrations. Then we performed titrations of Bst DNA polymerase and templates to confirm observations of inhibition by different dyes.

Several factors were considered for selecting the model LAMP systems. The performance of different fluorescent dyes in GPCR is dependent on the GC content of the amplified fragments and the optimal temperature of the reaction. For example, SYBR Green $I$ is known to bind preferentially to GC-rich sequences. In order to avoid any distortion of the results caused by peculiarities in a specific LAMP model system, we used three different DNA templates to evaluate the performance of various fluorescent dyes in qLAMP: lambda phage DNA, E. coli genomic DNA, and a genomic fragment of TBEV DNA (Table 1). The GC-content of the resulting amplicons ranged from $53.8 \%$ for E. coli genomic DNA to $57.8 \%$ for TBEV. On the other hand, the optimal reaction temperature was similar for lambda phage DNA and E. coli genomic 

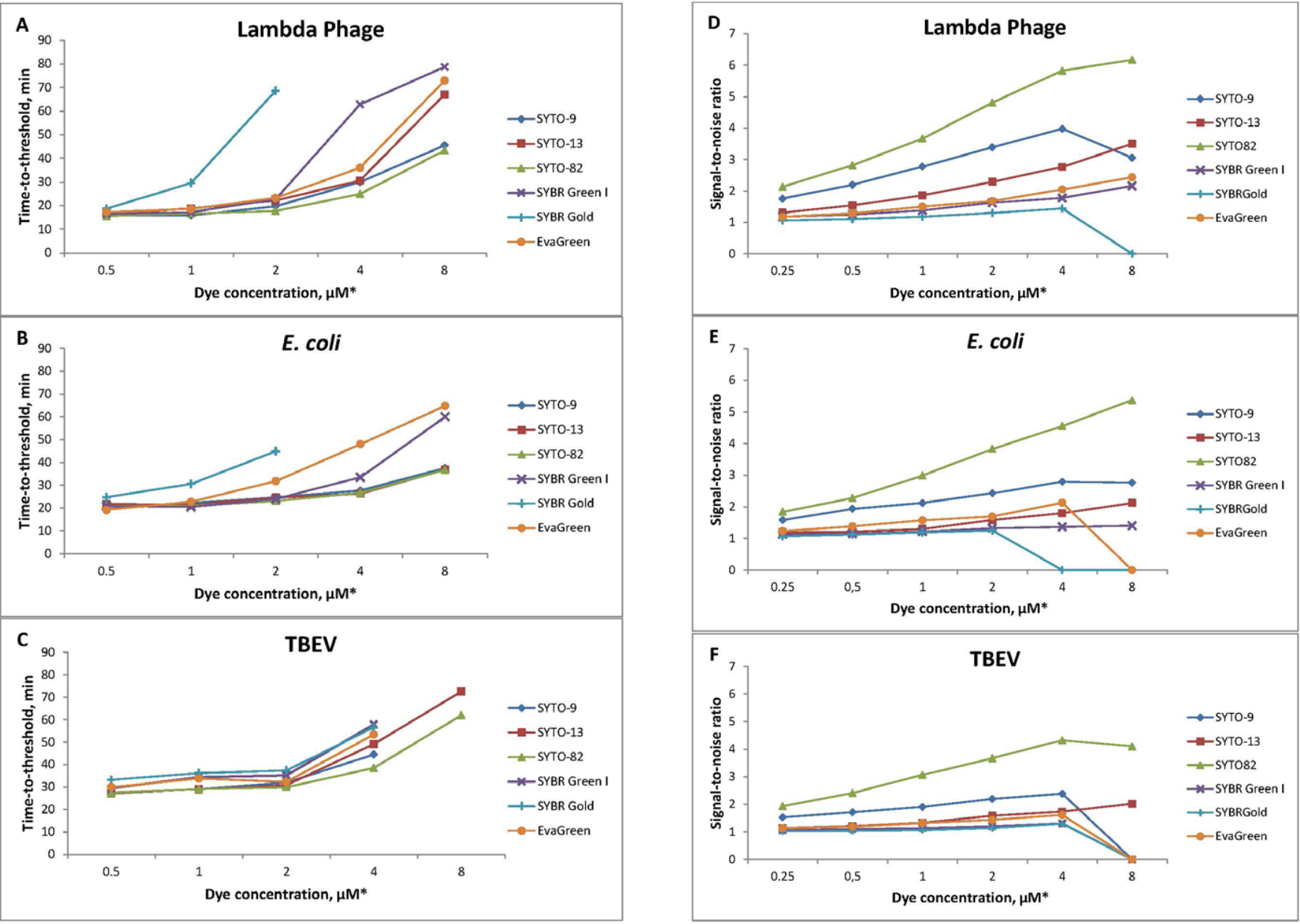

Figure 1. Serial dilution of fluorescent dyes in quantitative loop-mediated isothermal amplification (qLAMP). Six fluorescent dyes (SYTO-9, SYTO-13, SYTO-82, SYBR Green I, SYBR Gold, EvaGreen) were titrated in three model systems: lambda phage, E. coli, and tick-born encephalitis virus (TBEV). Stock solutions of dyes were prepared in MilliQ water; for qLAMP, dyes were titrated in the range of 0.25-8 $\mu \mathrm{M}$. qLAMP reactions for each dilution were run in triplicate. Diagrams represent the relationship of dye concentration to the time-to-threshold $\left(T_{+}\right)(A-C)$ or the signal-to-noise ratio (SNR) (D-F). The EvaGreen concentration is presented as fold-dilution because the molar concentration was not available (see the "Materials and methods" section).

DNA $\left(59^{\circ} \mathrm{C}\right.$ and $60^{\circ} \mathrm{C}$, respectively), while for TBEV the optimal temperature was lower $\left(57^{\circ} \mathrm{C}\right)$. Hence, the influence of both GC content and optimal reaction temperature was taken into account.

In qLAMP, $T_{\text {t }}$ can be used to indicate reaction efficiency, analogous to the quantification cycle $\left(C_{q}\right)$ reported for GPCR. $T_{t}$ values were plotted against dye concentration (Figure 1, A-C, Supplementary Table S1). Dye titration demonstrated that all of the analyzed intercalating fluorescent dyes can be used for qLAMP. As can be seen in Figure 1, the difference in fluorescence before and after the reaction was detectable, allowing the distinction of positive and negative reactions. However, the dyes inhibited qLAMP differently. Reaction efficiency decreases with increasing $T_{t}$, indicating an inhibitory effect. It should be noted that when the dye concentration is low, the $T_{t}$ also can be increased due to the extremely low change of fluorescence in the initial part of the reaction.

As would be expected, high dye concentrations resulted in high inhibition (Figure 1, A-C). The results obtained with different model systems were similar. An insignificant difference in the extent of inhibition between TBEV and the two other model systems (lambda phage and $E$. coli) can be caused by the higher GC content of the amplicon and the lower optimal reaction temperature for the TBEV LAMP system. The linear range of the reaction curves (Figure 1, A-C) was used to calculate the degree of inhibition. The slope of the line directly correlates with the degree of inhibition by the fluorescent dye (Table 2).
SYBR Gold inhibited the reaction the most, and SYTO-9 and SYTO-82 the least. SYBR Green I, EvaGreen, and SYTO-13 moderately decreased LAMP efficiency. These results are consistent with previous observations concerning the efficiency of qPCR with various fluorescent dyes: SYTO dyes (SYTO-9, SYTO-13, and SYTO-82) showed small inhibitory effects, while SYBR Green I negatively affected qPCR even at the recommended $1 \times(2 \mu \mathrm{M})$ concentration (17).

The SNR is an important characteristic that is defined as the ratio of the signal intensity to the noise intensity. A high SNR means a high reliability of the signal and a low probability of false-positive results. Here, we calculated the SNR as the ratio of fluorescence in the completed reactions with DNA template compared with the 

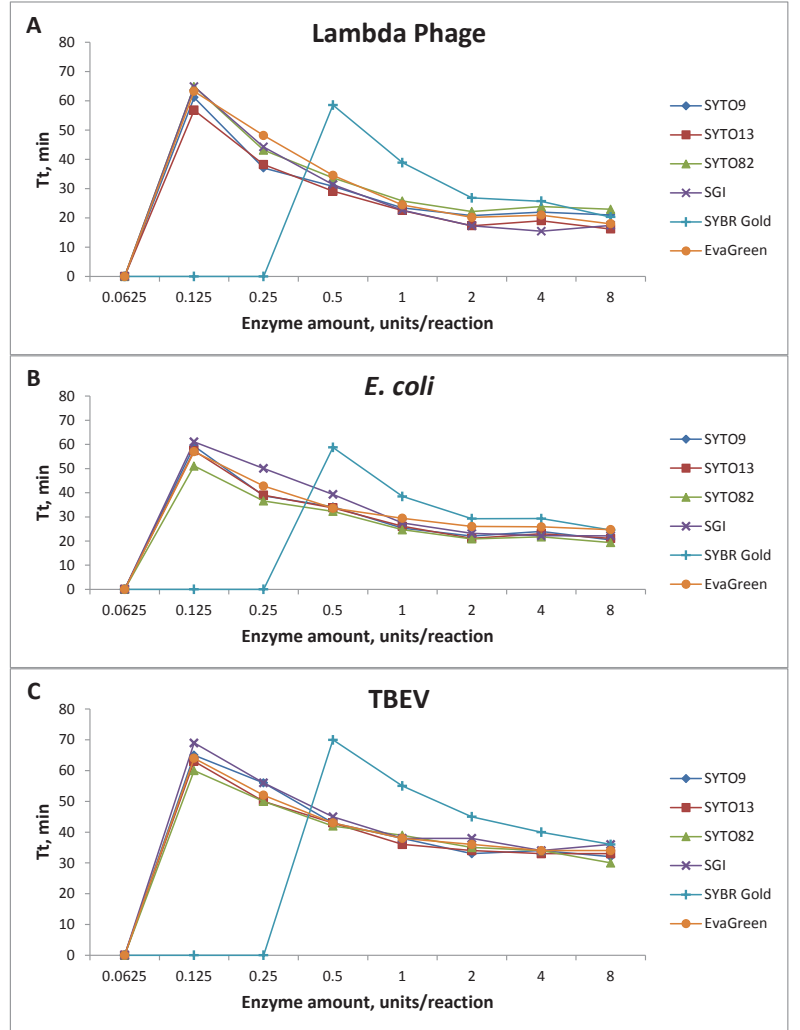

Figure 2. Quantitative loop-mediated isothermal amplification (qLAMP) with serial dilution of Bst DNA polymerase and different fluorescent dyes. Diagrams represent the relationship between the amount of enzyme and

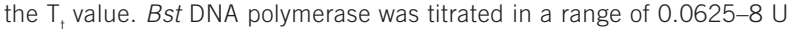
per reaction and used in 3 model systems: lambda phage (A), E. coli (B), and tick-born encephalitis virus (TBEV) (C). qLAMP reactions for each dilution were run in triplicate. The concentration of the fluorescent dyes in qLAMP was $1 \mu \mathrm{M}$ (except EvaGreen, $0.5 \times$, see Materials and Methods).

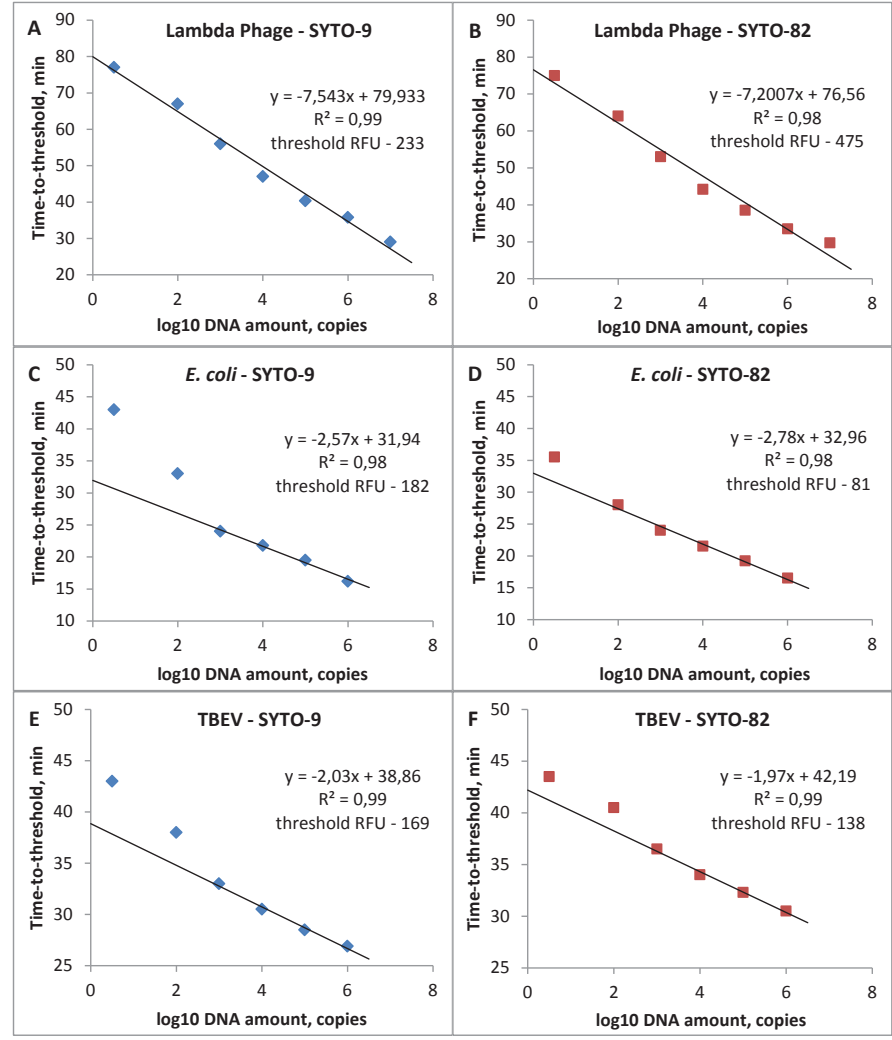

Figure 3. Serial dilution of template DNA in quantitative loop-mediated isothermal amplification (qLAMP) with dyes SYTO-9 and SYTO-82. Titration of lambda phage DNA, E. coligenomic DNA, and tick-born encephalitis virus (TBEV) DNA with SYTO-9 (A,C,E), and SYTO-82 $(B, D, F)$, respectively. Lambda phage DNA was diluted from $10^{7}$ to 5 copies per reaction; $E$. coli genomic DNA and TBEV DNA were diluted from $10^{6}$ to 5 copies per reaction; qLAMP for each dilution was don in triplicate. The concentration of the fluorescent dye in qLAMP was $1 \mu \mathrm{M}$. Assays were linear from $10^{6}$ to 5 template copies per reaction for lambda phage $(A, B)$ and from $10^{6}$ to $10^{3}$ template copies per reaction for $E$. coli $(C, D)$ and TBEV (E,F). no-template control. SYTO-82 demonstrated the best SNR at all concentrations tested, while the other fluorescent dyes had much lower SNRs. Among these, SYTO-9 had the highest SNR. The other four dyes could be divided into two groups where SYTO-13 and EvaGreen exhibited relatively higher SNRs, while SYBR Green I and SYBR Gold yielded the lowest SNRs.

The SNR depends directly on dye concentration; however, the inhibitory effect on amplification simultaneously increases. Therefore, for optimal performance, both the $T_{t}$ and the SNR should be considered in the selection of a fluorescent dye and its concentration for qLAMP. The best combination of low $T_{\text {t }}$ and high SNR was achieved with a dye concentration of $1 \mu \mathrm{M}(0.5 \times$ for EvaGreen), which was then used for further studies. Among the dyes, SYTO-82 demonstrated the highest suitability for qLAMP; SYTO-9 exhibited a similar $T_{t}$, but with a much lower SNR. The other fluorescent dyes
(SYTO-13, SYBR Green I, SYBR Gold, EvaGreen) showed higher $T_{t}$ and lower SNR values.

It should be noted that the stock 200 $\mu \mathrm{M}$ solutions of dyes were prepared with Milli-Q water without the addition of DMSO. This was done to minimize the effect of DMSO on qLAMP. Surprisingly, SYTO-13 was unstable under these conditions and became insoluble after a single freeze-thaw cycle, while the other dyes retained solubility.

We next tested the effect of Bst DNA polymerase concentration on qLAMP using the three model systems. It was observed that all of the examined fluorescent dyes were suitable with a low concentration of enzyme; however the reaction yield was different (Figure 2). The $T_{t}$ of the reactions with an equal amount of the enzyme was similar for almost all of the dyes. The only exception was SYBR Gold, which showed much stronger inhibition compared with the other dyes when using less than $4 U$ of enzyme. The other dyes can be used with enzyme amounts $>0.062 U$ per reaction, but $0.062 \mathrm{U}$ per reaction was insufficient to carry out qLAMP with all three model systems.

After comparing the degree of inhibition and the SNRs for all of the fluorescent dyes used in this study, SYTO-82 and SYTO-9 were chosen for further investigation since they demonstrated superior suitability for qLAMP. For the next stage, we carried out template titration. In the template titration experiments, we found the sensitivity of qLAMP with SYTO-9 and SYTO-82 was similar (Figure 3, Supplementary Figure S1). Both dyes enabled the detection of at least five copies of any of the model DNA templates per reaction. Assays were linear to 5 template copies per reaction for lambda phage and to $10^{3}$ template copies per reaction for $E$. coli and $T B E V$, using either SYTO-9 or SYTO-82.

We compared six fluorescent dyesSYTO-9, SYTO-13, SYTO-82, SYBR 
Table 2. Inhibition effect* of fluorescent dyes on qLAMP.

\begin{tabular}{|lccc|}
\hline \multicolumn{1}{|c|}{ Fluorescent dye } & Lambda Phage & $\begin{array}{c}\text { Model system } \\
\boldsymbol{E} \text {. coli }\end{array}$ & TBEV \\
\hline SYT0-9 & 7.35 & 4.72 & 5.51 \\
SYTO-13 & 11.28 & 3.48 & 6.75 \\
SYT0-82 & 6.39 & 3.73 & 3.41 \\
SYBR Green I & 17.02 & 9.12 & 8.6 \\
SYBR Gold & 24.95 & 10.05 & 7.14 \\
\hline EvaGreen & 12.88 & 11.64 & 6.87 \\
\hline * $T_{t}$ values from dye titration experiments were plotted against the dye concentration, and the linear ranges of \\
the resulting curves were used for further calculations of the line slopes, indicating the degree of inhibition. \\
\hline
\end{tabular}

Green I, SYBR Gold, EvaGreenconsidered suitable for quantitative LAMP. $T$, and SNR data were collected for different concentrations of the dyes and Bst DNA polymerase using three model systems. Among the dyes, SYTO-82 demonstrated the best combination of low inhibition and high SNR, allowing the rapid collection and interpretation of qLAMP data. Another dye, SYTO-9, exhibited low inhibition of qLAMP but had a lower SNR. The other dyes-SYTO-13, SYBR Green, SYBR Gold I, and EvaGreen-strongly inhibited qLAMP and had low SNRs.

\section{Author contributions}

U.A.B. and M.L.F. designed the study. I.P.O., E.A.B., and A.I.Z. performed the experiments and critically revised the data. I.P.O and M.L.F. wrote the manuscript.

\section{Acknowledgments}

This work was done with support from State Federal Budget Program No 62.2.3 and RAS program (I.30) FIMT 153.

\section{Competing interests}

The authors declare no competing interests.

\section{References}

1. Compton, J. 1991. Nucleic acid sequencebased amplification. Nature 350:91-92.

2. Fire, A., and S.Q. Xu. 1995. Rolling replication of short DNA circles. Proc Natl Acad Sci U S A. 92:4641-4645.

3. Notomi, T., H. Okayama, H. Masubuchi, T. Yonekawa, K. Watanabe, N. Amino, and T. Hase. 2000. Loop-mediated isothermal amplification of DNA. Nucleic Acids Res. 28:E63.

4. Wharam, S.D., P. Marsh, J.S. Lloyd, T.D. Ray, G.A. Mock, R. Assenberg, J.E. McPhee, P. Brown, et al. 2001. Specific detection of DNA and RNA targets using a novel isothermal nucleic acid amplification assay based on the formation of a three-way junction structure. Nucleic Acids Res. 29:E54.
5. Vincent, M., Y. Xu, and H. Kong. 2004. Helicase-dependent isothermal DNA amplification. EMBO Rep. 5:795-800.

6. Kurn, N., P. Chen, J.D. Heath, A. Kopf-Sill, K.M. Stephens, and S. Wang. 2005. Novel isothermal, linear nucleic acid amplification systems for highly multiplexed applications. Clin. Chem. 51:1973-1981.

7. Francois, P., M. Tangomo, J. Hibbs, E.-J. Bonetti, C.C. Boehme, T. Notomi, M.D. Perkins, and J. Schrenzel. 2011. Robustness of a loop-mediated isothermal amplification reaction for diagnostic applications. FEMS Immunol. Med. Microbiol. 62:41-48.

8. Mori, Y., K. Nagamine, N. Tomita, and T. Notomi. 2001. Detection of loop-mediated isothermal amplification reaction by turbidity derived from magnesium pyrophosphate formation. Biochem. Biophys. Res. Commun. 289:150-154.

9. Maeda, H., S. Kokeguchi, C. Fujimoto, I. Tanimoto, W. Yoshizumi, F. Nishimura, and S. Takashiba. 2005. Detection of periodontal pathogen Porphyromonas gingivalis by loopmediated isothermal amplification method. FEMS Immunol. Med. Microbiol. 43:233-239.

10. Veigas, B., R. Branquinho, J.V. Pinto, P.J. Wojcik, R. Martins, E. Fortunato, and P.V. Baptista. 2014. Ion sensing (EIS) real-time quantitative monitorization of isothermal DNA amplification. Biosens. Bioelectron. 52:50-55.

11. Goto, M., E. Honda, A. Ogura, A. Nomoto, and K.-I. Hanaki. 2009. Colorimetric detection of loop-mediated isothermal amplification reaction by using hydroxy naphthol blue. Biotechniques 46:167-172.

12. Tomita, N., Y. Mori, H. Kanda, and T. Notomi. 2008. Loop-mediated isothermal amplification (LAMP) of gene sequences and simple visual detection of products. Nat. Protoc. 3:877-882.

13. Tanner, N.A., Y. Zhang, and T.C. Evans. 2012. Simultaneous multiple target detection in real-time loop-mediated isothermal amplification. Biotechniques 53:81-89.

14. Chou, P.-H., Y.-C. Lin, P.-H. Teng, C.-L. Chen, and P.-Y. Lee. 2011. Real-time targetspecific detection of loop-mediated isothermal amplification for white spot syndrome virus using fluorescence energy transfer-based probes. J. Virol. Methods 173:67-74.

15. Tani, H., T. Teramura, K. Adachi, S. Tsuneda, S. Kurata, K. Nakamura, T. Kanagawa, and N. Noda. 2007. Technique for quantitative detection of specific DNA sequences using alternately binding quenching probe competitive assay combined with loop-mediated isothermal amplification. Anal. Chem. 79:5608-5613.
16. Aonuma, H., A. Yoshimura, T. Kobayashi, K. Okado, A. Badolo, B. Nelson, H. Kanuka, and S. Fukumoto. 2010. A single fluorescence-based LAMP reaction for identifying multiple parasites in mosquitoes. Exp. Parasitol. 125:179-183.

17. Gudnason, H., M. Dufva, D.D. Bang, and A. Wolff. 2007. Comparison of multiple DNA dyes for real-time PCR: effects of dye concentration and sequence composition on DNA amplification and melting temperature. Nucleic Acids Res. 35:e127.

18. Eischeid, A.C. 2011. SYTO dyes and EvaGreen outperform SYBR Green in real-time PCR. BMC Res. Notes 4:263.

19. Fischbach, J., N.C. Xander, M. Frohme, and J.F. Glökler. 2015. Shining a light on LAMP assays--a comparison of LAMP visualization methods including the novel use of berberine. Biotechniques 58:189-194.

20. Patel, J.C., J. Oberstaller, M. Xayavong, J. Narayanan, J.D. DeBarry, G. Srinivasamoorthy, L. Villegas, A.A. Escalante, et al. 2013. Real-time loop-mediated isothermal amplification (RealAmp) for the speciesspecific identification of Plasmodium vivax. PLoS One 8:e54986.

21. Tourlousse, D.M., F. Ahmad, R.D. Stedtfeld, G. Seyrig, J.M. Tiedje, and S.A. Hashsham. 2012. A polymer microfluidic chip for quantitative detection of multiple waterand foodborne pathogens using real-time fluorogenic loop-mediated isothermal amplification. Biomed. Microdevices 14:769-778.

22. Green, M.R. and J. Sambrook. 2012. Molecular Cloning: A Laboratory Manual (Fourth Edition). Cold Spring Harbor Laboratory Press, Cold Spring Harbor, NY.

23. Ahmed, M.U., S. Nahar, M. Safavieh, and M. Zourob. 2013. Real-time electrochemical detection of pathogen DNA using electrostatic interaction of a redox probe. Analyst 138:907-915

24. Hayasaka, D., K. Aoki, and K. Morita. 2013. Development of simple and rapid assay to detect viral RNA of tick-borne encephalitis virus by reverse transcription-loop-mediated isothermal amplification. Virol. J. 10:68.

25. Zipper, H., H. Brunner, J. Bernhagen, and F. Vitzthum. 2004. Investigations on DNA intercalation and surface binding by SYBR Green I, its structure determination and methodological implications. Nucleic Acids Res. 32:e103.

26. Mehndiratta, M., J.K. Palanichamy, P. Ramalingam, A. Pal, P. Das, S. Sinha, and P. Chattopadhyay. 2008. Fluorescence acquisition during hybridization phase in quantitative real-time PCR improves specificity and signal-to-noise ratio. Biotechniques 45:625-630.

Received 02 October 2015; accepted 15 March 2016.

Address correspondence to Igor P. Oscorbin, Institute of Chemical Biology and Fundamental Medicine, Siberian Branch of the Russian Academy of Sciences, Novosibirsk, Russian Federation. E-mail: osc.igor@gmail.com

To purchase reprints of this article, contact: biotechniques@fosterprinting.com 